\title{
On resilient behaviors in computational systems and environments
}

\author{
Vincenzo De Florio ${ }^{1}$
}

Received: 17 December 2014 / Accepted: 31 March 2015 / Published online: 1 May 2015

(C) Springer International Publishing Switzerland 2015

\begin{abstract}
The present article introduces a reference framework for discussing resilience of computational systems. Rather than a property that may or may not be exhibited by a system, resilience is interpreted here as the emerging result of a dynamic process. Said process represents the dynamic interplay between the behaviors exercised by a system and those of the environment it is set to operate in. As a result of this interpretation, coherent definitions of several aspects of resilience can be derived and proposed, including elasticity, change tolerance, and antifragility. Definitions are also provided for measures of the risk of unresilience as well as for the optimal match of a given resilient design with respect to the current environmental conditions. Finally, a resilience strategy based on our model is exemplified through a simple scenario.
\end{abstract}

Keywords Resilience - Elasticity - Computational antifragility $\cdot$ Behavior $\cdot$ Autonomic computing

\section{Introduction}

Resilience is one of those "general systems attributes" that appear to play a central role in several disciplines. Examples include ecology, business, psychology, industrial safety [32], microeconomics, computer networks, security, management science, cybernetics, control theory, as well as crisis and disaster management and recovery $[17,22,35,42,50]$. Although common traits are retained, in each discipline resilience takes

Vincenzo De Florio

vincenzo.deflorio@uantwerpen.be;

vincenzo.deflorio@gmail.com

1 MOSAIC Research Group, iMinds Research Institute, University of Antwerp, Middelheimlaan 1, 2020 Antwerp, Belgium peculiar domain-specific meanings [17]. To exacerbate the problem, even in the context of the same discipline often no consensus has been so far reached as to what are the peculiar aspects of resilience and what makes it different from, e.g., elasticity, dependability, or antifragility.

The present article contributes towards a solution to this problem in several ways. First, in Sect. 2, we introduce resilience, compare various of its domain-specific definitions, and derive a number of ancillary concepts and working assumptions. This allows resilience to be interpreted in Sect. 3 as the property emerging from the interaction of the behaviors exercised by a system and the environment it is set to operate in. The outcome of said interaction depends on both intrinsic and extrinsic factors: the "traits" of the system together with its endowment-the system's peculiar characteristics as well as its current state and requirements. At stake is the identity of the system, which we identify here with compliance to system specifications, including functional and non-functional system requirements.

The resilience interaction is modeled by considering the behaviors produced by the system and its environment. This provides us with a unifying framework within which it is possible to express coherent definitions of concepts such as elasticity, entelechism (change tolerance), and antifragility. Both system and environment are further modeled in terms of the "resilience organs" managing the five major services ancillary to resilience $[12,13]$ : perception, apperception, planning, executive, and knowledge management organscorresponding to the five modules of autonomic systems [30]. After this, in Sect. 4, we introduce measures of the optimality of a given resilient design with respect to the current environmental conditions: system supply and system-environment fit. One such strategy is detailed and exemplified in Sect. 5 through an ambient intelligence scenario. Finally, major results are recalled and final conclusions are drawn in Sect. 6. 


\section{Basic concepts}

The term "resilience" comes from Latin resilīre, "to spring back, start back, rebound, recoil, retreat", and is often intended and defined as the ability to cope with or recover from change. As mentioned in Sect. 1, this general definition has been specialized in different domains, in each of which it has taken domain-specific traits:

- In ecology, resilience often refers to an ecosystem's ability to respond to and recover from an ecological threat [27]. "Recovering" means here the ability to return to a steady state characterizing the ecosystem before the manifestation of the threat. The mentioned "steady state" represents the peculiar characteristics of the resilient ecosystem-its identity.

- In complex socioecological systems, resilience is the ability to absorb stress and maintain function in the face of climate change [23]. "Absorbing stress" clearly corresponds to the "recovering" ability found in ecology, though in this context the identity of the system lies in its function rather than in its state. An additional and peculiar aspect of resilient systems in this domain is given by their ability to improve systematically their sustainability.

- In organization science, (organizational) resilience is "the capacity to anticipate disruptions, adapt to events, and create lasting value" [3]. Here, the accents are on proactiveness and adaptation rather than on "springing back" to a past state or function. Intuitively, one may deduct that the former class of behaviors is more advanced than the latter one. One may also observe how in this case the definition brings to the foreground a fundamental component of system identity, namely the ability to create value.

- In social science and human ecology, resilience is "the ability of groups or communities to cope with external stresses and disturbances as a result of social, political and environmental change" [1]. Here, the recovery strategy of resilience is not made explicit. System identity implicitly refers to the identity of groups and communities and ranges from sociocultural aspects up to the ability to survive.

- In psychology, "resilience is the process of adapting well in the face of adversity, trauma, tragedy, threats or significant sources of stress [...] It means 'bouncing back' from difficult experiences" [4]. An important aspect here is the identification of resilience as a process: "Resilience should be considered a process, rather than a trait to be had" [39]; see also [24,34]. "System" identity is in the case of psychology the collection of beliefs about oneself.

- In material science, resilience is a material's property to "stay unaffected by an applied stress" up to some threshold, called "point of yielding", and to "return to its original shape upon unloading" [38]. Beyond the mentioned threshold, deformation is irreversible: "some residual strain will persist even after unloading" (ibid.) Here resilience is a property rather than a process, the key difference being the type of behavior exercised by the "systems". System identity is in this case represented by the shape or the characteristics of the material.

- In civil engineering, resilience is a construction's ability to absorb or avoid damage in the face of a natural or man-induced abnormal condition [29] such as flooding, hurricanes, or firepower. The considerations done in the case of material science apply also to this case.

- Finally, in computer science, resilience has been defined, e.g., as the ability to sustain dependability when facing changes [32]. This translates into the ability to avoid failure and at the same time the ability to sustain the delivery of trustworthy services. System identity is in this case as in the following definition.

Definition 1 (System identity) In the framework of artificial, computer-based systems, system identity is defined as a system's compliance to its system specifications and in particular to its functional and non-functional quality of service and quality of experience requirements.

As already remarked, with the change of the reference domain the above notions of resilience are applied to a spectrum of entities ranging from simple, passive-behaviored, individual objects to complex, teleological, collective adaptive systems. By making use of the behavioral approach introduced by Wiener et al. in [37], and briefly recalled in Sect. 3.1, in what follows three major classes in this spectrum are identified.

\subsection{Elastic objects and systems}

Resilience shall be referred to as elasticity when the system under consideration is only capable of simple types of behaviors: passive behavior and active, purposeful, nonteleological behaviors. In the former case, the system shall be referred to as an object.

The considerations made above with reference to, e.g., material science, apply also in this case. In particular, for both objects and servo-mechanisms resilience (elasticity) is represented as an intrinsic property: a trait.

Elastic systems able to exercise active behaviors are what Boulding refers to in [7] as "servo-mechanisms"- -systems whose action is predefined and is not modified by the interaction with other systems. In fact, servo-mechanisms do not "interact with other systems outside of themselves" [26] namely, they are not open systems.

Elastic systems and objects operate under the assumption that their environments are not going to exercise stress 
beyond their point of yielding. Quoting N. N. Taleb, they are system that "do not care too much" about their environments.

Another way to characterize elastic objects and systems is by observing that they have a predefined and static point of yielding. This introduces two syndromes, which we call "elastic undershooting" and "elastic overshooting."

\subsubsection{Elastic undershooting}

Elastic objects or systems are characterized at design time by a static yielding point beyond which they permanently lose their identity-for instance, they deformate; or break down; or fail; or become untrustworthy. The yielding point is therefore a resilience threshold. Whatever the characteristics of an elastic object or system, there is always a non-zero probability that the yielding point will be overcome.

Definition 2 (Elastic undershooting) When at time $t$ an elastic object or system with yielding point $Y$ is insufficiently resilient with respect to an experienced condition for which a yielding point $y(t)$ would be required, we shall call elastic undershooting (or simply undershooting, when this may be done without introducing ambiguity) the dynamic quantity $y(t)-Y$.

Undershooting is in fact as in a well-known fairy tale [28]: one may make their house of straw, of wood, or even of bricks; although more and more robust, each house will "just" shift the yielding point farther away; but that is all: there is no guarantee that, sooner or later, something stronger will show up and blow that house down, whatever the material it is made of. Development and operating costs, on the other hand, will grow up proportionally to the chosen yielding point-which brings us to the second syndrome.

\subsubsection{Elastic overshooting}

The choice of the yielding point represents the ability to cope with a worst-case scenario regardless of how frequently said condition will actually manifest itself. Unless the environmental conditions are deterministic and immutable, there will always be a non-zero probability that the yielding point is more pessimistic than what the experienced condition would require. In other words, an elastic system is prepared for the worst; but also it costs and expends resources as if the worst was actually there all the time.

Definition 3 (Elastic overshooting) When at time $t$ an elastic object or system with yielding point $Y$ is resilient with respect to an experienced condition for which a yielding point $y(t)$ would suffice, we shall call elastic overshooting (or simply overshooting, when this may be done without introducing ambiguity) the quantity $Y-y(t)$.
Overshooting reminds of the condition of the shell-snail that, "feeling always in danger of birds, lives constantly under its shield" [6, p. 147]—-thus carrying its weight at all times regardless of the actual presence or absence of birds.

\subsubsection{Observations}

Elastic undershooting and overshooting may be better understood when considering a well-known result by Shannon [45]: given an unreliable communication channel, it is always possible to transfer information reliably through it provided that a sufficient amount of information redundancy is foreseen. By means of the above-introduced terminology, Shannon's result may be formulated as follows: for any communication channel whose observed unreliability is $y(t)$ throughout a given time interval $T$, it is possible to define an elastic communication protocol with a yielding point

$Y>y(t) \forall t \in T$.

Ideally, the choice of $Y$ should be such that $Y$ represents the supremum of all the unreliability samples $y(t)$ observed during $T$. In this ideal case, no undershooting is experienced, although the system exhibits a cumulative overshooting equal to

$\int_{T} Y-y(t) \mathrm{d} t$

In more concrete situations in which unreliability drifting is unbound, undershooting would occur each time the value chosen for $Y$ would be less than the observed unreliability of the channel.

\subsection{System entelechies}

In Sect. 2, we have concisely reviewed a number of definitions of resilience emerged in the framework of diverse disciplines and domains. Several of such definitions explicitly require a resilient system to enact complex forms of behaviors: adapt reactively (see, e.g., in ecology and psychology) and adapt proactively (see, e.g., in organizational science). Such behaviors correspond respectively to simple teleological behaviors and extrapolative teleological behaviors (as defined in [37] and recalled in Sect. 3.1): behaviors that are driven respectively by the current state and by the hypothesized future state of an intended objective. Obviously, in this case, resilience cannot be regarded as a trait or attribute; rather, it is the emerging result of a process. Resilient systems are in motion to actively pursue the persistence of their system identity. The two just mentioned aspects correspond to the translation that Joe Sachs provides 
of the Aristotelian concept of entelechy1: "being-at-workstaying-itself" [40,41]. An entelechy is a system that is able to persist and sustain its completeness through a resilient process. "Completeness" here is to be intended as the characteristics that make of a system what it is: its "definition"-or, in other words, its system identity. Because of this, we shall refer to the systems in this resilience class as to entelechies.

The very nature of entelechies requires them to be able to "interact with other systems outside of themselves", namely to be open systems [26]. Such systems do not "want tranquility" nor expect their environments to be stable or stay the same. On the contrary, they assume conditions will vary, and adjust their function to the observed conditions or to speculated future conditions of their environments.

Another way to distinguish entelechies from elastic objects and systems is by observing that entelechies are characterized by dynamic and adaptive points of yielding. Undershootings and overshootings are still possible, though with a slightly different formulation:

Definition 4 (Entelechial undershooting) When, at time $t$, an entelechy with yielding point $Y(t)$ is insufficiently resilient with respect to an experienced condition for which a yielding point $y(t)$ would be required, we shall call entelechial undershooting (or simply overshooting, when this may be done without introducing ambiguity) the dynamic quantity $y(t)-Y(t)$.

Definition 5 (Entelechial overshooting) When, at time $t$, an entelechy with yielding point $Y(t)$ is resilient with respect to an experienced condition for which a yielding point $y(t)$ would suffice, we shall call entelechial overshooting (or simply overshooting, when this may be done without introducing ambiguity) the quantity $Y(t)-y(t)$.

An exemplary entelechy is given by an adaptive communication protocol for the reliable communication over an unreliable channel characterized by $y(t)$ unreliability. Such protocol would continuously "be at work" so as to estimate past and current values of $y(t)$ and extrapolate with them a future state $y\left(t^{\prime}\right)$. Once this speculated future value is known, the protocol would "stay itself" by choosing a yielding point $Y\left(t^{\prime}\right)$ as close as possible but still greater than $y\left(t^{\prime}\right)$.

More formally, the choice for $Y\left(t^{\prime}\right)$ would be such that

$0<Y\left(t^{\prime}\right)-\Pi\left(y\left(t^{\prime}\right)\right)<\varepsilon$,

where $\Pi\left(y\left(t^{\prime}\right)\right)$ represents a prediction of $y\left(t^{\prime}\right)$ and $\varepsilon>0$ expresses a safety margin to cover for inaccuracies in the prediction.

\footnotetext{
${ }^{1}$ Quoting Sachs, "Entelecheia means continuing in a state of completeness, or being at an end which is of such a nature that it is only possible to be there by means of the continual expenditure of the effort required to stay there." [40].
}

\subsection{Antifragile systems}

In the light of the discussion in Sects. 2.1 and 2.2, one may observe that most of the reported definitions of resilience correspond to either elastic objects / systems or to entelechies. An exception may be found in the class of complex socioecological systems. There we have systems that "are at work to stay themselves" (thus, they are entelechies), though are endowed with an additional feature: the ability "to improve systematically their sustainability". Wiener et al. did not explicitly consider behaviors including that ability [37]. The most closely related of their behavioral classes—one could say its genus proximum [9]-is given by teleological behaviors.

As discussed in more detail in Sect. 3.1, teleological systems are those characterized by a feedback loop: their behavior

"is controlled by the margin of error at which the [system] stands at a given time with reference to a relatively specific goal" [37].

Due to its purely behavioral nature, the approach followed in the cited work does not cover organizational, architectural, and structural aspects. Because of this, no account is given on the modifications that a teleologically behaviored system would apply to itself to achieve its goal.

At least the following four cases may occur:

1. The feedback loop is purely exogenous: the system action is simply steered towards the goal (in its current or hypothesized future position.)

2. The feedback loop is both exogenous and endogenous. Internal changes only concerns the "knobs", namely the parameters of the system. This case corresponds to parametric adaptation.

3. The feedback loop is both exogenous and endogenous; the internal changes adapt the structure of the system. This corresponds to system reconfigurations (namely structural adaptation). Adaptations are phenotypical and do not affect the identity of the system. Furthermore, the experience leaves no trace on the identity of the system.

4. The feedback loop is both exogenous and endogenous; the internal changes adapt any of the following aspects: the function; the structure; the architecture; and the organization of the system. Changes are genotypical: they are persisted and modify permanently the nature of the system.

It is important to remark that, while in cases 1-3 the system is "at work to stay itself" $[40,41]$, in case 4 the system is "at work to get better". At least in the case of complex socioecological systems, teleological behaviors belong to this fourth category: through their experience, those systems elaborate a 
feedback that is also endogenous and affects the genotypical ability "to improve systematically their sustainability". The feedback thus affects the identity of the system. Rather than adapting, the system evolves.

In the case of complex socioecological systems, said evolution leads to an improvement in sustainability: those systems "enhance the level of congruence or fit between themselves and their surroundings" [47]. This matches the concept introduced by Taleb in [48]: antifragile systems. Quoting from the cited reference, "Antifragility is beyond resilience or robustness. The resilient resists shocks and stays the same; the antifragile gets better."

In what follows, we distinguish explicitly this class of teleological behaviors and systems by referring to them as to antifragile systems, which we define as follows:

Definition 6 (Antifragile system) We shall call a system "antifragile" if it is able to exercise teleological behaviors that evolve the system and its identity in such a way as to systematically improve the fit with their environment.

By considering the just enunciated definition, we can observe that

- Antifragile systems are not necessarily more resilient than entelechies or elastic objects and systems. As it is the case for those entities, also antifragile systems are characterized by a yielding point - a resilience threshold beyond which they would fail; break down; or become untrustworthy.

- Antifragile systems mutate their system identity. By referring to Definition 1, this means that the behaviors of antifragile computer-based systems may drift outside of what prescribed in their specifications. Scenarios such as those that Stephen Hawking [25] and many others [33] are warning of become more concrete when considering this particular characteristic of antifragile systems.

- Antifragile systems must possess some form of awareness of their current and past system-environment fit; in particular, they must be able to create and maintain a model of the risk of losing one or more aspects of their system identity.

Going back to the communication protocol presented in Sect. 2.2, an exemplary antifragile system would be a protocol that, in addition to being able to estimate quantity $y\left(t^{\prime}\right)$, also learns how to mutate its own algorithm so as to profit from the characteristics of the environment. As an example, instead of sending, say, $Y$ redundant copies for each of the packets of its messages, the protocol could realize that a better strategy (with respect to the current behavior of the channel) would be that of interleaving the transmission of packets of different messages. This would result in a more efficient strategy such that a higher yielding point would be reached with a consumption of resources lower than in the original algorithm.

At the same time, it is important to observe how the introduced interleaving would affect several peculiar characteristics of the protocol-for instance, it would introduce jitter (viz., a drifting in the periodicity of the messages). Repercussions on the validity of the specifications become then possible. For instance, if the protocol were intended for a teleconferencing service, the introduced jitter would affect the quality of experience of the users of that service. Embedding the same protocol in a service insensible to periodicity drifting (such as a file transferring service) would not translate in a loss of system identity.

\subsection{A few observations}

As a summary of the discussion in this section, we can derive here a number of observations:

\subsubsection{Resilience is a relative figure}

As observed in [16], resilience is a dynamic property whose emergence is influenced by at least the following two factors:

1. The intrinsic characteristics of the system: in particular, whether the system is elastic, entelechial, or antifragile.

2. The extrinsic "level of congruence or fit between [the system] and [its] surroundings" [47].

The first factor is absolute in nature and tells how "evolved" the class of the system is. The second factor is a relative one, and tells how the system's behavior is able to match the conditions currently expressed by the environment. This second factor makes of resilience a relative figure. Whatever a system's structure, organization, architecture, capabilities, and resources, that system is resilient only so long as its implementation matches the conditions currently exercised by the environment ${ }^{2}$.

\subsubsection{Resilience is the product of an interplay between a system and its environment}

As a corollary to what mentioned in Sect. 2.4.1, we observe that resilience is not a property, but rather the product of a $a$ process. Such process corresponds to the dynamic interplay between two entities: a system and its environment.

\footnotetext{
2 Possibly, the first Scholar to have distinguished intrinsic and extrinsic factors towards the emergence of resilience was von Leibniz [15].
} 


\subsubsection{The environment is a system}

"Environment" is interpreted here and in what follows simply as another system; in particular, as a collective system (in other words, a "system-of-systems") taking different shapes, including for instance any combination of cyberphysical things; biological entities such as human beings; servo-mechanisms; and intelligent ambients able to exercise complex teleological behaviors.

\subsubsection{Resilience is an interplay of behaviors}

Resilience is one of the possible outcomes of an interplay of behaviors. If and only if the interplay between the system behaviors and the environmental behaviors is one that preserves the system identity then the system will be called resilient. As discussed in Sect. 3.1, behaviors may range from the random behaviors typical of electromagnetic sources up to the "intelligent", cybernetic behaviors characterizing, e.g., human beings and complex ambient environments.

\subsection{Preliminary conclusions}

A major conclusion here - and a starting point for the treatise in next section - is given by the intuitive notion that evaluating resilience must be done not merely considering a system's intrinsic characteristics; rather, it should be done by expressing in some convenient form the dynamic fit between the system and its environment. This may be obtained, e.g., by comparing the resilience class of the system with the dynamically mutating resilience class of the environment. Another, more detailed method could be by comparing the behaviors of system and environment. Yet another approach could be to apply the behavioral comparison method to specific organs of the system and its environment-for instance those organs that are likely to play a significant role in the emergence of resilience or its opposite.

In what follows, we focus on the last mentioned approach.

\section{Resilient behaviors, organs, and methods}

To proceed with the present treatise, we first recall in Sect. 3.1 the major classes of behaviors according to the classic discussion by Rosenblueth, Wiener, and Bigelow [37]. After this, in Sect. 3.2, five major services that play a key role towards the emergence of resilience are identified. Finally, in Sect. 3.3, we use the concepts introduced so far to reformulate definitions for elasticity, entelechism, and antifragility.

\subsection{Behavioral classification}

As already mentioned, Rosenblueth, Wiener, and Bigelow introduced in [37] the concept of the "behavioristic study of natural events", namely "the examination of the output of the object and of the relations of this output to the input"3. The term "object" in the cited paper corresponds to that of "system". In that renowned text, the authors purposely "omit the specific structure and the intrinsic organization" of the systems under scrutiny and classify them exclusively on the basis of the quality of the "change produced in the surroundings by the object", namely the system's behavior. The authors identify in particular four major classes of behaviors ${ }^{4}$ :

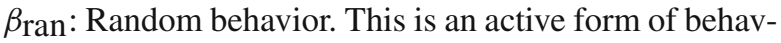
ior that does not appear to serve a specific purpose or reach a specific state. A source of electromagnetic interference exercises random behavior.

$\beta_{\text {pur: }}$ Purposeful, non-teleological behavior. This is behavior that serves a purpose and is directed towards a specific goal. In purposeful behavior, a "final condition toward which the movement [of the object] strives" can be identified. Servo-mechanisms provide an example of purposeful behavior.

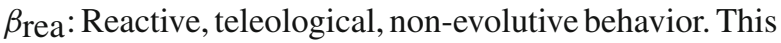
is behavior that "involve[s] a continuous feedback from the goal that modifies and guides the behaving object". Examples of this behavior include phototropism, namely the tendency that can be observed, e.g., in certain plants, to grow towards the light, and gravitropism, viz., the tendency of plant roots to grow downwards. As already mentioned (see Sect. 2.2), reactive behaviors require the system to be open [26] (i.e., able to continuously perceive, communicate, and interact with external systems and the environment) and to embody some form of feedback loop. Class $\beta_{\text {rea }}$ is non-evolutive, meaning that the experienced change does not influence the identity of the system (cf. Sect. 2.3).

$\beta$ pro: Proactive, teleological, non-evolutive behavior. This is behavior directed towards the extrapolated future state of the goal. The authors in [37] classify proactive behavior according to its "order", namely the amount of context variables taken into account in the course of the extrapolation. As class $\beta_{\text {rea, }}$, so class $\beta_{\text {pro }}$ is nonevolutive.

By considering the arguments in Sect. 2.3, a fifth class can be added:

$\beta_{\text {ant }}$ : teleological evolutive behaviors. This is the behavior emerging from antifragile systems (see Sect. 2.3 for more detailed on this class of systems).

\footnotetext{
${ }^{3}$ If not otherwise specified the quotes in the present section are from [37].

${ }^{4}$ For the sake of brevity passive behavior shall not be discussed here.
} 
Each of the above five classes may see their systems operate in isolation or through some form of social interaction. To differentiate these two cases, we add the following attribute:

$\sigma(b)$ : True when $b$ is a social behaviors. This attribute identifies behaviors based on the social interaction with other systems deployed in the same environment. Examples of such behaviors include, among others, mutualistic, Commensalistic, parasitic, co-evolutive, and co-opetitive behaviors [5,8]. For more information, the reader is referred to Boulding's discussion in his classic paper [7] and, for a concise survey of social behaviors, to [17].

The resilience classes introduced in Sect. 2 can now be characterized in terms of the above behavioral classes: elastic systems correspond to $\beta_{\text {pur }}$; non-evolving entelechies exercise either $\beta$ rea or $\beta$ pro behaviors; while, as already mentioned, $\beta$ ant pertains to antifragile systems.

We shall define $\pi$ as a projection map returning, for each of the above behavior classes, an integer in $\{1, \ldots, 5\}$ $\left(\pi\left(\beta_{\text {ran }}\right)=1, \ldots, \pi\left(\beta_{\text {ant }}\right)=5\right)$. Aim of $\pi$ is twofold: it associates an integer "identifier" to each behavioral class and it introduces an "order" among classes. Intuitively, the higher is the order of class, the more complex is the behavior.

In what follows, it is assumed that behaviors manifest themselves by changing the state of measurable properties. As an example, behavior may translate into a variation in the electromagnetic spectrum perceived as a change in luminosity or color. Context figures are the term that shall be used to refer to those measurable properties.

For any behavior $\beta_{x}$ dependent on a set of context figures $F$, notation $\beta_{x}^{F}$ will be used to denote that $\beta_{x}$ is exercised by considering the context figures in $F$. Thus if, for instance, $F=$ (speed, luminosity), then $\beta_{\text {rea }}^{F}$ refers to a reactive behavior that responds to changes in speed and light.

For any behavior $\beta_{x}$ and any integer $n>0$, notation $\beta_{x}^{n}$ will be used to denote that $\beta_{x}$ is exercised by considering $n$ context figures, without specifying which ones.

As an example, behavior $\beta_{\text {pro }}^{|F|}$, with $F$ defined as above, identifies an order- 2 proactive behavior, while $\beta_{\text {pro }}^{F}$ says in addition that that behavior considers both speed and luminosity to extrapolate the future position of the goal.

Now, the concept of partial order among behaviors is introduced.

Definition 7 (Partial order of behaviors) Given any two behaviors $\beta_{1}$ and $\beta_{2}, \beta_{1} \prec \beta_{2}$ if and only if either of the following conditions holds:

1. $\left(\pi\left(\beta_{1}\right) \leq \pi\left(\beta_{2}\right)\right) \wedge\left(\exists(F, G): \beta_{1}=\beta_{1}^{F} \wedge \beta_{2}=\beta_{2}^{G} \wedge F\right.$ $\subsetneq G$ ). In other words, $\beta_{1} \prec \beta_{2}$ if (1) $\beta_{1}$ belongs to a behavioral class that is at most equal to $\beta_{2}$ 's (via function $\pi)$ and (2) $\beta_{2}$ is based on a set of context figures that extends $\beta_{1}$ 's.

2. $\left(\pi\left(\beta_{1}\right) \leq \pi\left(\beta_{2}\right)\right) \wedge\left(\exists(F, G): \beta_{1}=\beta_{1}^{|F|} \wedge \beta_{2}=\beta_{2}^{|G|} \wedge\right.$ $F \subsetneq G)$.

This case is equivalent to case 1 , the only difference being in the notation o the behavior.

3. $\left(\pi\left(\beta_{1}\right)=\pi\left(\beta_{2}\right)\right) \wedge\left(\sigma\left(\beta_{1}\right)=\right.$ false $) \wedge\left(\sigma\left(\beta_{2}\right)=\right.$ true $)$. In other words, $\beta_{1} \prec \beta_{2}$ also when both $\beta_{1}$ and $\beta_{2}$ belong to the same behavioral class, though $\beta_{2}$ is a social behavior while $\beta_{1}$ is not.

For any two resilient systems $p_{1}$ and $p_{2}$, respectively, characterized by behavioral classes $\beta_{1}$ and $\beta_{2}$, if $\beta_{1} \prec \beta_{2}$ then $p_{1}$ is said to exhibit "systemically inferior" resilience with respect to $p_{2}$.

It is important to observe that $\prec$ is about the intrinsic resilience characteristics of the system (see Sect. 2.4.1). Partial order $\prec$ does not tell which system is "more resilient"; it highlights that for instance system "dog" is able to exercise behaviors that are less complex than those of system "man". This tells nothing about the extrinsic "level of congruence or fit" [47] that for instance a "man" or a "dog" may exhibit in a given environment. As exemplified, e.g., in [14], when a threat is announced by ultrasonic noise, a "dog" able to perceive the threat and flee could result more resilient than a "man". The use of sentinel species [44] is in fact a social behavior based on this fact. An application of this principle is given in Sect. 5 .

\subsection{Resilience organs}

As done in [16], it is conjectured here that reasoning about a system's resilience is facilitated by considering the behaviors of the system organs that are responsible for the following abilities:

M: the ability to perceive change;

A: the ability to ascertain the consequences of change;

P: the ability to plan a line of defense against threats deriving from change;

$\mathbf{E}$ : the ability to enact the defense plan being conceived in step $\mathbf{P}$;

$\mathbf{K}$ : and, finally, the ability to treasure up past experience and systematically improve, to some extent, abilities $\mathbf{M}$, $\mathbf{A}, \mathbf{P}$, and $\mathbf{E}$.

These abilities correspond to the components of the so-called MAPE-K loop of autonomic computing [30]. In the context of the present paper, the system components responsible for those abilities shall be referred to as "resilience organs".

The following notation shall be used to refer to organ $\mathbf{O}$ of system $s$ : $s . \mathbf{O}$ (for $\mathbf{O} \in\{\mathbf{M}, \ldots, \mathbf{K}\}$ ). 
Definition 8 (Cybernetic class) For any system $s$, the 5-tuple corresponding to the behaviors associated to its resilience organs,

$(s . \mathbf{M}, s . \mathbf{A}, s . \mathbf{P}, s . \mathbf{E}, s . \mathbf{K})$,

shall be referred to as the cybernetic class of $s$.

Two observations are important for the sake of our discussion.

Observation 1 (Intrinsic resilience) A system's cybernetic class puts to the foreground how intrinsically resilient that system is (see again Sect. 2.4.1) and makes it easier to compare whether certain resilience organs (or the whole system) are (resp., is) systemically inferior to (those of) another system.

As an example, the adaptively redundant data structures described in [21] have the following cybernetic class:

$\mathcal{C}_{1}=\left(\beta_{\text {pur }}, \beta_{\text {pro }}^{1}, \beta_{\text {pur }}, \beta_{\text {pur }}, \emptyset\right)$,

while the adaptive $N$-version programming system introduced in $[10,11]$ is

$\mathcal{C}_{2}=\left(\beta_{\text {pur }}, \beta_{\text {pro }}^{2}, \beta_{\text {pur }}, \beta_{\text {pur }}, \beta_{\text {pur }}\right)$

By comparing the above 5 -tuples $\mathcal{C}_{1}$ and $\mathcal{C}_{2}$ one may easily realize how the major strength of those two systems lies in their analytic organs, both of which are capable of proactive behaviors $\left(\beta_{\text {pro }}\right)$ - though in a simpler fashion in $\mathcal{C}_{1}$. Another noteworthy difference is the presence of a knowledge organ in $\mathcal{C}_{2}$, which indicates that the second system is able to accrue and make use of the past experience to improve- to some extent-its action ${ }^{5}$.

Please note that not all the behaviors introduced in Sect. 3.1 may be applied to all of a system's organs. For instance, it would make little sense to have a perception organ behave randomly (unless one wants to model, e.g., the effect of certain hallucinogenic substances in chemical warfare ${ }^{6}$.)

Observation 2 (Extrinsic resilience) A system's cybernetic class puts to the foreground also how extrinsically resilient that system is if the above comparison is done between the

\footnotetext{
$\overline{5}$ The presence of a knowledge organ does not mean that its system is antifragile. In the case at hand, for instance, the system does not mutate it system identity-it stays an $N$-version programming system.

${ }^{6}$ See for instance the interview with Dr. James S. Ketchum, in the Sixties a leading psychiatrist at the Army Chemical Center at Edgewood Arsenal in Maryland, US: "With BZ [3-quinuclidinyl benzilate], the individual becomes delirious, and in that state is unable to distinguish fantasy from reality, and may see, for instance, strips of bacon along the edge of the floor." [46].
}

cybernetic class of the system and the dynamically evolving cybernetic class of the environment (see Sect. 2.4.1). This comparison represents a system-environment fit, in turn indicating the property emerging from the interplay between the current state of the system and the current state of the environment-in other words, the system is likely to either preserve or lose its peculiar features because of the interaction with the environment. In the former case, the system shall be called as "resilient;" in the second case, "unresilient."

\subsection{Again on elasticity, entelechism, and antifragility}

The model and approach introduced thus far provide us with a conceptual framework for alternative definitions of elasticity, entelechism, and antifragility — namely the resilience classes introduced in Sect. 2.

Definition 9 (Elasticity) Given a system $s$ and its cybernetic class $\mathcal{C}_{s}$, with $s$ deployed in environment $e, s$ shall be called "elastic" with respect to $e$ if $s$ is resilient (in the sense expressed in Observation 2) and if the behaviors in $\mathcal{C}_{s}$ are purposeful ( $\left.\beta_{\text {pur }}\right)$ and defined, once and for all, at design or deployment time.

Elasticity corresponds to simple, static behaviors that make use of a system's predefined internal characteristics and resources so as to mask the action of external forces. Those characteristics and resources take the shape of various forms of redundancy which is used to mask, rather than tolerate, change.

Definition 10 (Entelechism) Given a system $s$ and its cybernetic class $\mathcal{C}_{s}$, with $s$ deployed in environment $e, s$ shall be referred to as "entelechy" with respect to $e$ if $s$ is resilient (in the sense expressed in Observation 2) and if the behaviors corresponding to the $\mathbf{A}$ and $\mathbf{P}$ organs of $\mathcal{C}_{s}$ are either of type $\beta_{\text {rea }}$ or $\beta$ pro. Entelechism, or change tolerance, is defined as the property exhibited by an entelechy.

As evident from their definition, entelechies are open, context-aware systems; able to autonomically repair their state in the face of disrupting changes; and able to guarantee their system identity. A knowledge organ may or may not be present and, depending on that, the feedback organs may or may not be stateful—meaning that "memory" of the experienced changes is or is not retained.

Definition 11 (Computational antifragility) Given a system $s$ and its cybernetic class $\mathcal{C}_{s}$, with $s$ deployed in environment $e$, we shall say that $s$ is computationally antifragile with respect to $e$ when the following conditions are all met:

1. The awareness organ of $s, \mathbf{A}$, is open to the systemenvironment fit between $s$ and $e$. In particular, as suggested in Sect. 2.3, this means that A implements a model 
of the risk of losing one or more aspects of the system identity of $s$. One such model is exemplified, e.g., by the distance-to-failure function introduced in [19] and discussed in [12].

2. Throughout time intervals in which the behavior of $e$ is stable, the planning organ $\mathbf{P}$ is able to monotonically improve extrinsic resilience (see Observation 2) and thus optimize risk/performance trade-offs.

3. Organ $\mathbf{P}$ evolves through machine learning or other machine-oriented experiential learning [31], leading $s$ to evolve towards ever greater intrinsic (systemic) resilience (see Obs. 1).

4. Organ $\mathbf{K}$ is stateful and persists lessons learned from the experience and its "conceptualization."

Computationally antifragile systems are system-environment fit-aware systems; able to embody and persist systemic improvements suggested by the match of the current system identity with the current environmental conditions. The learning activity possibly implies a 4-stage cycle similar to the one suggested by Kolb in [31], executed concurrently with the resilience behaviors of the $\mathbf{M}, \mathbf{A}, \mathbf{P}$, and $\mathbf{E}$ organs.

\section{Approach}

As already mentioned, a methodological assumption in the present article is that the evolution of an environment may be expressed as a behavior. Said behavior may be of any of the types listed in Sects. 3.1 and 3.3 and it may result in the dynamic variation of a number of "firing context figures". In fact, those figures characterize and, in a sense, set the boundaries of an ecoregion, namely "an area defined by its environmental conditions" [2]. An environment may be the behavioral result of the action of, e.g., a human being (a "user"); or the software agents managing an intelligent ambient; or for instance it may be the result of purposeless (random) behavior - such as a source of electromagnetic interference. As a consequence, an environment may for instance behave (or appear to behave) randomly, or it may exhibit a recognizable trend; in the latter case the variation of its context figures may be such that it allows for tracking or speculation (extrapolation of future states). Moreover, an environment may exhibit the same behavior for a relatively long period of time or it may vary frequently its character.

Given an environment (or a system), the dynamic evolution of the environmental (resp. systemic) behavior shall be referred to as "turbulence". Diagrams such as the one in Fig. 1 may be used to represent the dynamic behavioral evolution of either environments or systems.

Whenever two behaviors $\beta_{1}$ and $\beta_{2}$ are such that $\beta_{1} \prec \beta_{2}$, it is possible to define some notion of distance between the two behaviors. One way to define such "behavioral metric

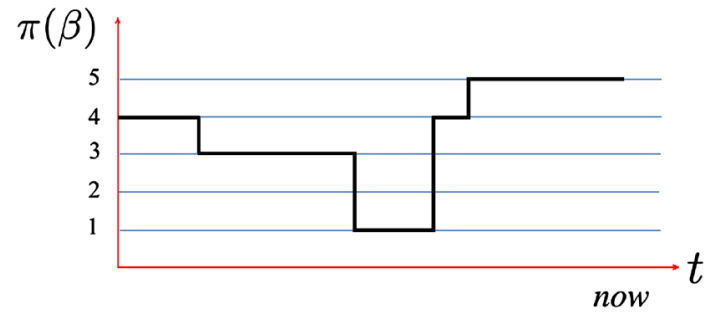

Fig. 1 Exemplification of turbulence, namely the dynamic evolution of environmental or systemic behavior. Abscissas represent time, "now" being the current time. Ordinates are the behavior classes exercised by either the environment or the system

function" would be by encoding the characteristics of each behavior onto the bits of a binary word, with the three most significant bits encoding the projection map of the behavior and the bits from the fourth onward encoding the cardinality of the set of context figures or the order of the behavior. When the behaviors belong to the same class although considering two different context sets, say $F$ and $G$, then a simpler formulation of a distance would be $\mathbf{a b s}(|F|-|G|)$. Let us call dist one such metric function.

It is now possible to propose a definition of two indicators for the extrinsic quality of resilience: The system supply relative to an environment and the system-environment fit.

Definition 12 (System supply) Let us consider a system $s$ deployed in an environment $e$, characterized respectively by behaviors $\beta^{s}(t)$ and $\beta^{e}(t)$. Let us assume that $\beta_{1}$ and $\beta_{2}$ are such that either $\beta_{1} \prec \beta_{2}$ or $\beta_{2} \prec \beta_{1}$. Given a behavioral metric function dist defined as above, the following value shall be called as supply of $s(t)$ with respect to $\beta^{e}(t)$ :

$$
\begin{aligned}
& \operatorname{supply}(s, e, t) \\
& = \begin{cases}\boldsymbol{d i s t}\left(\beta^{s}(t), \beta^{e}(t)\right) & \text { if } \beta^{e}(t) \prec \beta^{s}(t) \\
-\boldsymbol{d i s t}\left(\beta^{s}(t), \beta^{e}(t)\right) & \text { if } \beta^{s}(t) \prec \beta^{e}(t) \\
0 & \text { if } \beta^{e}(t) \text { and } \beta^{s}(t) \\
& \text { express the same behaviors. }\end{cases}
\end{aligned}
$$

Supply can be positive (referred to as "oversupply"), negative ("undersupply"), or zero ("perfect supply").

Observation 3 Oversupply and undersupply provide a quantitative formulation of the notions of overshooting and undershooting given in Sect. 2.

Definition 13 (System-environment fit) Given the same conditions as in Definition 12, the following function:

$\boldsymbol{f i t}(s, e, t)= \begin{cases}1 /(1+\operatorname{supply}(s, e, t)) & \text { if } \operatorname{supply}(s, e, t) \geq 0 \\ -\infty & \text { otherwise. }\end{cases}$

shall be referred to as "system-environment fit of $s$ and $e$ at time $t . "$ 


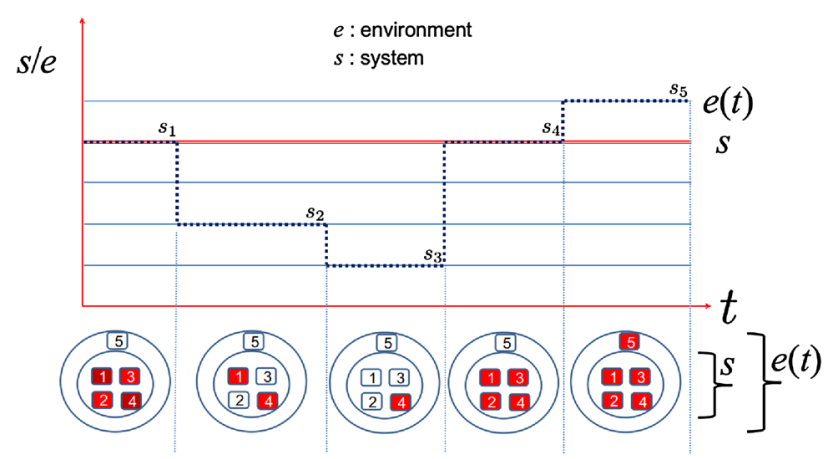

Fig. 2 Exemplification of supply and system-environment fit

The above definition expresses system-environment fit as a function returning 1 in the case of best fit; slowly scaling down with oversupply; and returning $-\infty$ in case of undersupply. The reason for the infinite penalty in case of undersupply is due to the fact that it signifies an undershooting or, in other words, a loss of system identity.

The just enunciated formulation is of course not the only possible one: an alternative one could be, for instance, by having supply ${ }^{2}$ instead of supply in the denominator of fit in Definition 13. Another formulation could extend optimal fit to a limited region of oversupply as a safety margin to cover for inaccuracies in the prediction of the behavior of the environment. This is similar to the role of $\varepsilon$ in (1).

Figure 2 exemplifies a system-environment fit in the case of two behaviors $\beta^{s}$ and $\beta^{e}$ with $s \subsetneq e$. Environment $e$ affects five context figures identified by integers $1, \ldots, 5$ while $s$ affects context figures $1, \ldots, 4$. The system behavior is assumed to be constant, thus for instance if $s$ is a perception organ then it constantly monitors the four context figures $1, \ldots, 4$. On the contrary, $\beta^{e}$ varies with time. Five time segments are exemplified $\left(s_{1}, \ldots, s_{5}\right)$ during which the following context figures are affected:

$s_{1}$ : Context figures $1, \ldots, 4$.

$s_{2}$ : Context figure 1 and context figure 4 .

$s_{3}$ : Context figure 4.

$s_{4}$ : Context figures $1, \ldots, 4$.

$s_{5}$ : Context figures $1, \ldots, 5$.

Context figures are represented as boxed integers, with an empty box meaning that the figure is not affected by the behavior of the environment and a filled box meaning the figure is affected. The behavior of the environment is constant within a time segment and changes at the next one. This is shown through the sets at the bottom of Fig. 2: for each segment $t_{s} \in\left\{s_{1}, \ldots, s_{5}\right\}$ the superset is $e\left(t_{s}\right)$ while the subset is $s\left(s_{t}\right)$, namely $e\left(s_{t}\right) \cap s$. The relative supply and the system-environment fit also change with the time segments. During $s_{1}$ and $s_{4}$ there is perfect supply and best fit: the behav- ior exercised by the environment is evenly matched by the features of the system. During $s_{2}$ and $s_{3}$, we have overshootings: the systemic features are more than enough to match the current environmental conditions. It is a case of oversupply. Correspondingly, fit is rather low. In $s_{5}$, the opposite situation takes place: the systemic features-for instance, pertaining to a perception organ-are insufficient to become aware of all the changes produced by the environment. In particular, here changes associated with context figure 5 go undetected. This is a case of undersupply (that is to say, undershooting), corresponding to a loss of identity: the "worst possible" system-environment fit.

\subsection{Supply- and fit-aware behaviors}

Whenever a partial order " $\prec$ " exists between a system's and its environment's behaviors, it is possible to consider system behaviors of the following forms:

1. Either $b=\beta_{\text {pro }}^{F}$ or $b=\beta_{\text {ant }}^{F}$, with $\sigma(b)=$ false and with $F$ including figures that provide a measure of the risk of unresilience, expressed, e.g., through supply and fit.

Such behavior corresponds to condition 11 in Definition 11 , namely one of the necessary conditions to computational antifragility. When exercised by system organs for analysis, planning, and knowledge management, this behavior translates in the possibility to become aware of and speculate about the possible future resilience requirements. If this is coupled with the possibility to revise one's system organs by enabling or disabling, e.g., the ability to perceive certain context figures depending on the extrapolated future environmental conditions, then a system could use this behavior to improve proactively its own system-environment fit—possibly mutating its features and functions.

An exemplary system based on this feature is given by the already mentioned adaptively redundant data structures of [21] and the adaptive $N$-version programming system introduced in $[10,11]$. Those systems make use of the so-called reflective variables [20] to perceive changes in a "distance-to-failure" function. Such function basically measures the probability of failure of a voting scheme at the core of the replication strategies adopted in the mentioned systems. In other words, such function estimates the probability of undersupply for voting-based software systems.

2. Behavior $b$ defined as in case 1 , but with $\sigma(b)=$ true. In this case, the analysis, planning and knowledge organs are aware of other systems in physical or logical proximity and may use this fact to artificially augment or reduce their system features by establishing / disestablishing 
mutualistic relationships with neighboring systems. An example of this strategy is sketched in Sect. 5.

Note how both behaviors 1 and 2 may evolve the system beyond its current identity. In case 2 , the behavior augments the social "scale" of the system, which becomes a part of a greater whole - in other words, a resilient collective system [18].

As a final remark, we observe how the formulation of system-environment fit adopted in the present article may be augmented so as to include other factors-for instance, overheads and costs.

\section{Scenario}

In the present section, the approach introduced in Sect. 4 is exemplified through an ambient scenario. As we did in [13], also our scenario here is inspired by the use of the so-called sentinel species [44], namely systems or animals able to compensate for another species' insufficient perception. We now introduce the main actors in our scenario.

\subsection{Coal Mine}

Our ambient is called "Coal Mine". Reason for this name is to highlight how the behavior of this ambient may randomly change from a neutral state (NS) to a threatening state (TS), as it occasionally occurs in "real-life" coal mines when high concentrations of toxic substances-e.g., carbon monoxide and dioxide, or methane-manifest themselves. (Toxic gases in high concentration are lethal to both animals and human beings.)

By making use of the terminology introduced in Sects. 3.1 and 3.2, we shall refer to the behaviors of Coal Mine as to $\beta_{C M}=\beta_{\text {ran }}^{T}$, where $T$ represents a context set including a figure, let us call it $t$, telling whether Coal Mine is in its neutral or threatening state.

Several systems may be deployed in Coal Mine. Let us call Miner one such system.

\subsection{Miner}

Miner is a system whose intrinsic resilience (see Observation 1) is very high: Miner's resilience organs are capable of advanced behaviors, including perception of a wide range of context figures; proactive analysis and planning; and a knowledge organ able to persist lessons learned from experience.

In particular, let us refer to $\beta_{M}$ as to the behavior of the perception organ of the Miner (that is to say, Miner.M). Let us assume $\beta_{M}$ to be a purposeful behavior able to report changes in some set $F$ of context figures. In other words, $\beta_{M}=\beta_{\text {pur }}^{F}$.
In what follows, we assume $T \backslash\{t\} \subsetneq F$, and $t \notin F$. Those assumptions mean that Miner.M can become aware of any type of changes in Coal Mine, with the exception of a NSto-TS transition. Miner is thus unable to perceive the threat and therefore it is unresilient with respect to Coal Mine.

\subsection{Canary}

Let us now suppose, we have a second system called "Canary". Canary's organs are all intrinsically inferior (cf. Observation 1) with respect to Miner's, with the exception of its perception organ, Canary.M. Let us call $\beta_{C}$ the behavior of Canary.M. In what follows, we assume $\beta_{C}$ to be equal to $\beta_{\text {pur }}^{G}$ for some set $G$ of context figures. In addition, we assume that both $F \subsetneq G$ and $G \subsetneq F$ are false. Miner.M and Canary.M are thus incommensurable - none of the conditions in Definition 7 apply: neither Miner.M $\prec$ Canary.M nor Canary.M $\prec$ Miner.M is true.

\subsection{Discussion}

The advanced features of the analysis, planning, and knowledge organs of Miner allowed it to deduct two relevant facts:

1. $t \in G$. In other words, despite its comparably simpler nature, Canary can detect a NS-to-TS transition in Coal Mine-what in a "physical" coal mine would represent a dangerous increase in the concentration of toxic gases.

2. Canary is more susceptible than Miner [36] to the persistence of the TS state in Coal Mine. In other words, when deployed in Coal Mine in its threatening state, Canary is likely to experience general failures (what we could refer to as "total losses of the system identity") much sooner than Miner.

Miner thus realizes that, by bringing along instances of the Canary system and by monitoring for their condition and failures, it may artificially augment its perception organ. This technique is known as biomonitoring [49]. The new collective system Miner+Canary is now characterized by a perception organ whose behavior is of type $\beta_{\mathrm{pur}}^{F \cup G}$. Let us refer to Miner+Canary as to MC.

Now, as $t \in G$, it follows that $T \subsetneq F \cup G$. This matches one of the conditions in Definition 7, thus $\beta_{\mathrm{CM}} \prec \beta_{\mathrm{MC} . \mathbf{M}}$. Behaviors are now commensurable, and it is possible to deduct that Coal Mine now exhibits "systemically inferior" resilience with respect to the monitoring organ of MC.

It is now possible to define a strategy based on Miner and multiple instances of Canary. 
Fig. 3 Estimations of supply and fit when 100 instances of Canary are used. Abscissas represent the number of failed Canary instances

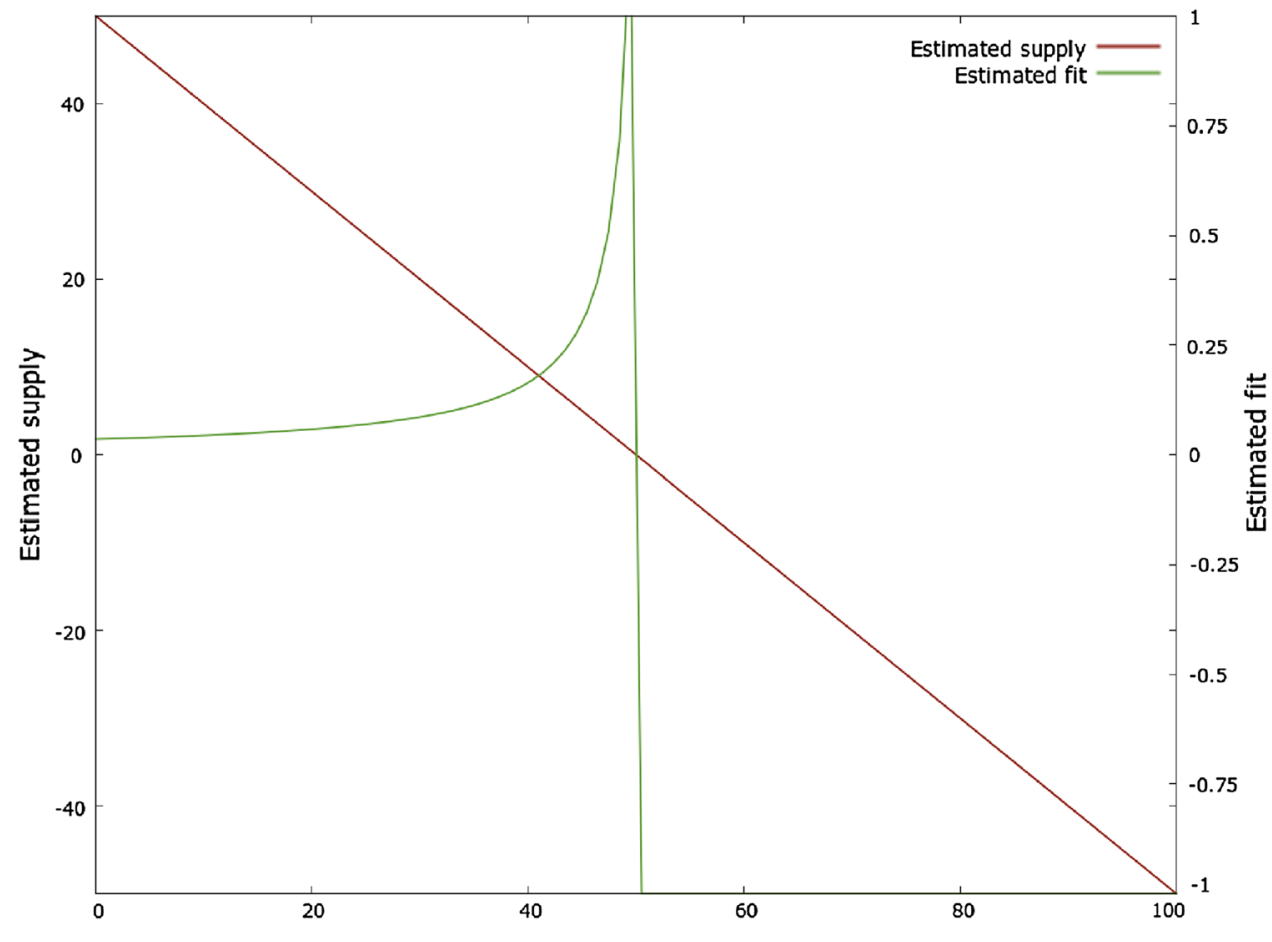

\subsection{Strategy}

First, we estimate the supply of Miner.M with respect to Coal Mine. The estimation is based on a probabilistic assessment of the distance between the two involved behaviors. Said assessment is formulated by considering the amount of Canary replicas that have failed out of a predefined maximum equal to $|c|$ :

\section{float EstimateSupply (Coal Mine $\mathrm{cm}$, Miner $m$, Canary $c[]$ )} Begin

1 int $f=0$

2 Query state of the Canary instances $c$ deployed in $\mathrm{cm}$;

3 For each failed Canary in $c$, increment $f$;

4 return $|c| / 2.0-f$;

End

Second, through the estimated supply we can derive an estimated fit as follows:

float EstimateFit (Coal Mine $\mathrm{cm}$, Miner $m$, Canary $c[$ ])

\section{Begin}

1 float supply = EstimateSupply $(\mathrm{cm}, m, c[])$;

2 if supply $\geq 0$ then return $1 /(1+$ supply $)$;

3 else return FLOAT_MIN;

\section{End}

By executing a function like EstimateFit, Miner evolves into a collective system by means of a $\beta_{\text {Soc }}$ behavior. Said behavior augments the system's social scale, embedding the system into a greater "whole". In this case, the established social relationship is parasitic rather than mutualistic, as it enhances the resilience of one of the partners at the expenses of the other one (Fig. 3).

\subsection{Final remarks}

The just described scenario is clearly an exemplary simplification. A full fledged example would see a much more complex confrontation of behaviors of different organs and at different system scales. A possible conceptual framework for modeling such "behavioral confrontations" may be found in Evolutionary Game Theory [43] (EGT). In EGT, the system and its deployment environment could be modeled as opponents that confront behavioral strategies of the classes discussed in this paper. This interpretation matches particularly well cases where either or both of the environment and the system explicitly aim at threatening their opponent, as it is typical of certain security scenarios.

As a last remark, we observe how detecting incommensurability between a system's behavior and its environment's provides the system with awareness of the need to establish a social relationship - a new behavior $b$ such that $\sigma(b)=$ true - such as a mutualistic or a parasitic relationship.

\section{Conclusions}

This paper discusses resilience as the behavior resulting from the coupling of a system and its environment. Depending 
on the interactions between these two "ends" and on the quality of the individual behaviors that they may exercise, resilience may take the form of elasticity (change masking); entelechism (change tolerance); or antifragility (adapting to and learning from change). Following the lesson of Leibniz [15], resilience is decomposed in this paper into an intrinsic and an extrinsic component- the former representing the static, "systemic" aspects of a resilience design, the latter measuring the contingent match between that design and the current environmental conditions. It is conjectured that optimal resilience may be more easily attained through behaviors that are not constrained by the hard requirement of preserving the system identity. Such "antifragile behaviors" are exemplified through a scenario in which a system establishes a parasitic relationship with a second system to artificially augment its perception capability. Finally, we observe how several of the concepts discussed in this paper match well with corresponding concepts in EGT. In particular, the choice of which behavior to enact corresponds with the choice of a strategy; resilience is the outcome of an interplay - a "game"; and the interplay between the "players" (system and environment, as well as their organs) translates in penalties and rewards. Because of those similarities it is conjectured here that a possible framework for the design of optimal resilience strategies may be given through EGT, by modeling both system and environment as two opponents choosing behavioral strategies with the explicit purpose to "win" the adversary. In this new model, we shall distinguish between a system's behavior and a system's manifested behavior. The former is what we have focused on in this paper and characterizes the "systemic class" of the system-what the system is capable to do. The latter is the behavior the system decides to manifest; it is a "move" in a confrontation between two opponents. Thus for instance an intelligent agent able to exercise advanced behaviors may decide to behave (pseudo-)randomly so as to, e.g., confuse the opponent, or even to cause the opponent choose a yielding point and then use this information to "attack" it and lead it to an undershooting. Future work will include proposing one such model and assessing its benefits.

Acknowledgments I would like to acknowledge the valuable suggestions of the reviewers, which allowed me to correct many inaccuracies and improve considerably the coherence of this work. Last but far from the least I would like to thank my wife, father-in-law, and son, for providing me with the environment, the time, and the tranquility for me to concentrate on this article for several hectic weeks.

\section{References}

1. Adger WN (2000) Social and ecological resilience: are they related? Prog Hum Geogr 24(3):347-364. http://phg.sagepub.com/ content $/ 24 / 3 / 347$
2. Anonymous (2014) Collins English Dictionary-Complete \& Unabridged, 10th edn. HarperCollins Publishers. http://dictionary. reference.com/browse/ecoregion

3. Anonymous (2015) Resiliency measurement required. Tech. rep., Resilience Corporation . http://resilient.com/wp-content/uploads/ 2015/01/Resilience-Measurement-Required-January2015

4. Anonymous (2015) The road to resilience. Retrieved on 21 Mar 2015 from http://www.apa.org/helpcenter/road-resilience.aspx

5. Astley W, Fombrun CJ (1983) Collective strategy: social ecology of organizational environments. Acad Manag Rev 8:576-587

6. Bayley RS (1836) Nature considered as a revelation. Hamilton, Adams, and Co, London. https://archive.org/details/ natureconsidere00baylgoog

7. Boulding K (1956) General systems theory-the skeleton of science. Manag Sci 2(3):197-208

8. Brandenburger A, Nalebuff B (1998) Co-opetition-a revolutionary mindset that combines competition and cooperation. Currency Doubleday, New York

9. Burek P (2004) Adoption of the classic theory of definition to ontology modeling. In: Bussler C, Fensel D (eds) Proceedings of the 11th Int.1 Conf. on Artificial Intelligence: methodology, systems, and applications (AIMSA 2004), LNAI 3192. Springer

10. Buys J, De Florio V, Blondia C (2011) Towards context-aware adaptive fault tolerance in soa applications. In: Proceedings of the 5th ACM International Conference on Distributed Event-Based Systems (DEBS), pp 63-74. Association for Computing Machinery, Inc. (ACM). doi:10.1145/2002259.2002271

11. Buys J, De Florio V, Blondia C (2012) Towards parsimonious resource allocation in context-aware n-version programming. In: Proceedings of the 7th IET System Safety Conference. The Institute of Engineering and Technology

12. De Florio V (2013) On the constituent attributes of software and organisational resilience. Interdiscip Sci Rev 38(2). http:// www.ingentaconnect.com/content/maney/isr/2013/00000038/ 00000002/art00005

13. De Florio V (2013) Preliminary contributions towards autoresilience. In: Proceedings of the 5th International Workshop on Software Engineering for Resilient Systems (SERENE 2013). Lecture Notes in Computer Science vol 8166, Springer, Kiev, Ukraine, pp 141-155

14. De Florio V (2013) What system is the most resilient? http:// eraclios.blogspot.be/2013/11/what-system-is-most-resilient. html. (Posted on ERACLIOS: Elasticity, Resilience, Antifragility in CoLlective and Individual Objects and Systems)

15. De Florio V (2014) Behavior, organization, substance: three gestalts of general systems theory. In: Proceedings of the IEEE 2014 Conference on Norbert Wiener in the 21st Century. IEEE

16. De Florio V (2014) On the behavioral interpretation of systemenvironment fit and auto-resilience. In: Proceedings of the IEEE 2014 Conference on Norbert Wiener in the 21st Century. IEEE

17. De Florio V (2014) Quality Indicators for Collective Systems Resilience. Emerg Complex Org 16(3):65-104

18. De Florio V (2015) Communication and control: tools, systems, and new dimensions, chap. Reflections on organization, emergence, and control in sociotechnical systems. Lexington. https:// arxiv.org/abs/1412.6965

19. De Florio V, Blondia C (2007) Reflective and refractive variables: a model for effective and maintainable adaptive-and-dependable software. In: Proceedings of the 33rd Euromicro Conference on Software Engineering and Advanced Applications (SEEA 2007), Software Process and Product Improvement track (SPPI). IEEE Computer Society, Lübeck, Germany

20. De Florio V, Blondia C (2007) Reflective and refractive variables: A model for effective and maintainable adaptive-and-dependable software. In: Proceedings of the 33rd EUROMICRO Conference on 
Software Engineering and Advanced Applications (SEAA 2007). Lübeck, Germany

21. De Florio V, Blondia C (2008) On the requirements of new software development. Int J Bus Intell Data Min 3(3):330-349

22. De Florio V, Sun H, Blondia C (2014) Community resilience engineering: reflections and preliminary contributions. In: Majzik I, Vieira M (eds) Software Engineering for Resilient Systems. Lecture Notes in Computer Science, vol 8785, pp 1-8. Springer International Publishing. doi:10.1007/978-3-319-12241-0_1

23. Folke C (2006) Resilience: the emergence of a perspective for social-ecological systems analyses. Global Environ Change 16:253-267. doi:10.1016/j.gloenvcha.2006.04.002

24. Gilligan R (2001) Promoting resilience: a resource guide on working with children in the care system. British Agencies for Adoption and Fostering London (2001)

25. Hawking S, Russell S, Tegmark M, Wilczek F (2014) Stephen Hawking: Transcendence looks at the implications of artificial intelligence-but are we taking AI seriously enough? Indipendent. http://goo.gl/U2nBMJ

26. Heylighen F (1998) Basic concepts of the systems approach. In: Heylighen F, Joslyn C, Turchin V (eds) Principia Cybernetica Web. Principia Cybernetica, Brussels. http://pespmc1.vub.ac.be/ SYSAPPR.html

27. Holling C (1973) Resilience and stability of ecological systems. Ann Rev Ecol Syst 4:1-23. doi:10.1146/annurev.es.04.110173. 000245

28. Jacobs J (1890) English Fairy Tales. David Nutt, London. https:// archive.org/details/englishfairytal00jacogoog

29. Jennings BJ, Vugrin ED, Belasich DK (2013) Resilience certification for commercial buildings: a study of stakeholder perspectives. Environ Syst Decis 33(2):184-194. doi:10.1007/ s10669-013-9440-y

30. Kephart JO, Chess DM (2003) The vision of autonomic computing. Computer 36:41-50. doi:10.1109/MC.2003.1160055

31. Kolb D (1984) Experiential learning: experience as the source of learning and development. Prentice Hall, Englewood Cliffs, NJ

32. Laprie JC (2008) From dependability to resilience. In: IEEE/IFIP International Conference on Dependable Systems and Networks (DSN 2008-Fast Abstracts). http://2008.dsn.org/fastabs/ dsn08fastabs_laprie

33. Future of Life Institute (2015) Research priorities for robust and beneficial artificial intelligence: an open letter. http://futureoflife. org/misc/open_letter

34. Masten AS (1994) Resilience in individual development: successful adaptation despite risk and adversity. In: Wang M, Gordon E (eds) Risk and resilience in inner city America: challenges and prospects. Erlbaum, Hillsdale, pp 3-25

35. Meyer JF (2009) Defining and evaluating resilience: a performability perspective. In: Proceedings of the International Workshop on Performability Modeling of Computer and Communication Systems (PMCCS)
36. Rabinowitz PM, Scotch ML, Conti LA (2010) Animals as sentinels: Using comparative medicine to move beyond the laboratory. ILAR J 51(3):262-267. doi:10.1093/ilar.51.3.262 http:// ilarjournal.oxfordjournals.org/content/51/3/262.abstract

37. Rosenblueth A, Wiener N, Bigelow J (1943) Behavior, purpose and teleology. Philos Sci 10(1):18-24. http://www.journals.uchicago. edu/doi/abs/10.1086/286788

38. Roylance D (2001) Mechanics of materials. Wiley, Somerset, New Jersey. http://goo.gl/ruf7us

39. Rutter M (2008) Developing concepts in developmental psychopathology. In: Hudziak J (ed) Developmental psychopathology and wellness: Genetic and environmental influences. American Psychiatric Publishing, Washington DC, pp 3-22

40. Sachs J (2015) Aristotle: motion and its place in nature. In: Fieser J, Dowden B (eds) Internet encyclopedia of philosophy. Retrieved on 22 Mar 2015 http://www.iep.utm.edu/aris-mot/

41. Sachs J (1995) Aristotle's Physics: a guided study. Masterworks of discovery. Rutgers University Press, New Brunswick, NJ

42. Salles RM, Marino DA (2011) Strategies and metric for resilience in computer networks. Comput J. doi:10.1093/comjn1/ bxr110 http://comjnl.oxfordjournals.org/content/early/2011/10/ 19/comjnl.bxr110.abstract

43. Sandholm WH (2012) Evolutionary game theory. In: Meyers RA (ed) Computational Complexity: Theory, Techniques, and Applications. Springer, New York, pp 1000-1029

44. van der Schalie WH, Gardner HS, Bantle JA, Rosa CTD, Finch RA, Reif JS, Reuter RH, Backer LC, Burger J, Folmar LC, Stokes WS (1999) Animals as sentinels of human health hazards of environmental chemicals. Environ Health Perspect 107(4). http://www. ncbi.nlm.nih.gov/pmc/articles/PMC1566523

45. Shannon CE, Winer AD, Sloane NJA (1993) Claude Elwood Shannon: Collected Papers. Wiley, New York, NY

46. Sirius KGR (2007) Hallucinogenic weapons: the other chemical warfare. http://www.podtrac.com/pts/redirect.mp3?rusiriusradio. com/shows/rusirius-088.mp3

47. Stokols D, Lejano RP, Hipp J (2013) Enhancing the resilience of human-environment systems: a social ecological perspective. Ecol Soc 18(1):7

48. Taleb NN (2012) Antifragile: things that gain from disorder. Random House Publishing Group, New York

49. Tingey DT (1989) Biologic markers of air-pollution stress and damage in forests. The National Academies Press, Washington, DC. http://goo.gl/P5BmIv

50. Trivedi K, Kim DS, Ghosh R (2009) Resilience in computer systems and networks. In: Computer-Aided Design-Digest of Technical Papers, 2009. ICCAD 2009. IEEE/ACM International Conference on, pp 74-77 